\title{
Blood Speckle Imaging Compared with Conventional Doppler Ultrasound for Transvalvular Pressure Drop Estimation in an Aortic Flow Phantom
}

Cameron Dockerill ( $\square$ cameron.dockerill@kcl.ac.uk)

King's College London https://orcid.org/0000-0003-0153-3389

Harminder Gill

King's College London

Joao F. Fernandes

King's College London

Amanda Q.X. Nio

King's College London

Ronak Rajani

King's College London

Pablo Lamata

King's College London

\section{Research Article}

Keywords: Blood speckle imaging, Doppler, Ultrasound, Echocardiography, Transvalvular pressure drop, Aortic stenosis

Posted Date: January 25th, 2022

DOI: https://doi.org/10.21203/rs.3.rs-1292561/v1

License: (c) (1) This work is licensed under a Creative Commons Attribution 4.0 International License. Read Full License 
1 Title: Blood Speckle Imaging Compared with Conventional Doppler

2 Ultrasound for Transvalvular Pressure Drop Estimation in an Aortic Flow

3 Phantom

4

5 Authors: Cameron Dockerill*, Harminder Gill*, Joao F. Fernandes* , Amanda Q. X. Nio*,

6 Ronak Rajani $^{* \dagger}$ and Pablo Lamata*

7 Author affiliations: *School of Biomedical Engineering \& Imaging Sciences, King's College

8 London, London, United Kingdom.

$9 \quad \dagger G u y ’ s$ and St Thomas’ NHS Foundation Trust, London, United Kingdom.

10 Corresponding author: Professor Pablo Lamata, School of Biomedical Engineering \&

11 Imaging Sciences, King's College London, London, United Kingdom.

12 Email: pablo.lamata@kcl.ac.uk

14 Key words: Blood speckle imaging; Doppler; Ultrasound; Echocardiography; Transvalvular pressure drop; Aortic stenosis

Word count: 3,308 


\section{Abstract}

Background Transvalvular pressure drops are assessed using Doppler echocardiography for the diagnosis of heart valve disease. However, this method is highly user-dependent and may overestimate transvalvular pressure drops by up to $54 \%$. This work aimed to assess transvalvular pressure drops using velocity fields derived from blood speckle imaging (BSI), as a potential alternative to Doppler.

Methods A silicone 3D-printed aortic valve model, segmented from a healthy CT scan, was placed within a silicone tube. A CardioFlow 5000MR flow pump was used to circulate blood mimicking fluid to create eight different stenotic conditions (transvalvular flow velocities between 1.6 and $2.4 \mathrm{~m} / \mathrm{s}$ and pressure drops between 9.7 and $22.7 \mathrm{mmHg}$ ). Eight PendoTech pressure sensors were embedded along the tube wall to record ground-truth pressures (10 $\mathrm{KHz}$ ). The Bernoulli equation with measured probe angle correction was used to estimate pressure drop from maximum velocity values acquired across the valve using Doppler and BSI with a GE Vivid E95 ultrasound machine and 6S-D cardiac phased array transducer.

Results There were no significant differences between pressure drops estimated by Doppler, BSI and ground-truth at the lowest stenotic condition $(10.4 \pm 1.76,10.3 \pm 1.63$ vs. $10.7 \pm$ $1.12 \mathrm{mmHg}$, respectively; $\mathrm{p}>0.05$ ). Significant differences were observed between the pressure drops estimated by the three methods at the greatest stenotic condition $(26.4 \pm 1.52$, $14.5 \pm 2.14$ vs. $20.1 \pm 1.78 \mathrm{mmHg}$ for Doppler, BSI and ground-truth, respectively; $\mathrm{p}<0.05$ ). Across all conditions, Doppler overestimated pressure drop (Bias $=3.54 \mathrm{mmHg}$, while BSI underestimated pressure drop $($ Bias $=-3.68 \mathrm{mmHg})$.

Conclusions BSI accurately estimated pressure drops only up to $10.7 \mathrm{mmHg}$ in controlled phantom conditions of low stenotic burden. Although BSI offers a number of theoretical advantages to conventional Doppler echocardiography, further refinements and clinical 
studies are required with BSI before it can be used to improve transvalvular pressure drop estimation in the clinical evaluation of aortic stenosis.

\section{$48 \quad$ Background}

Doppler echocardiography is routinely used in clinical practice to assess the severity of aortic stenosis. The maximum velocity of blood flow through the aortic valve during systole is recorded, and the simplified Bernoulli equation is used to estimate the transvalvular pressure drop (a more accurate term to the widely used gradient) across the valve [1]. This technique is preferred to cardiac catheterisation as it is non-invasive, widely accessible and inexpensive [2].

Despite this, pressure drop estimation using Doppler echocardiography is highly userdependent and has been shown to overestimate transvalvular pressure drops by up to 54\% [3]. Taking a single peak velocity event ignores the complex haemodynamics of blood flow across the valve, where the full velocity profile at the point of maximum flow constriction is needed to estimate the actual pressure drop. In addition, if the angle of insonation is not fully aligned with the direction of blood flow, the maximum velocity will be missed [4]. Several noninvasive alternatives have been studied but are not yet applied clinically [5].

Blood speckle imaging (BSI) has recently emerged as an alternative methodology for the assessment of aortic stenosis severity [6]. By the direct measurement and visualisation of blood vector velocity fields $[7,8]$ it has the potential to overcome some of the limitations with conventional Doppler echocardiography. BSI utilises existing technology from tissue speckletracking that is commonly used to evaluate myocardial deformation [9]. A small image kernel is defined in the first image of the vessel and the same speckle signature is tracked in the following frame. This is then repeated for a grid of measurements to quantify the velocity and direction of the blood flow [10]. Acquiring blood flow velocity data in this way is advantageous 
as it potentially allows pressure drop to be calculated from velocity data across 3-dimensions, rather than from a single streamline in conventional Doppler echocardiography. The principal aim of the current manuscript was to evaluate blood speckle imaging (BSI) and Doppler echocardiography for pressure drop estimations against ground-truth pressure sensors in a bespoke aortic phantom with a 3D-printed aortic valve at various flow rates.

\section{Materials and Methods}

\section{Pressure Drop Phantom}

In order to investigate the accuracy and utility of novel techniques for pressure drop estimation, a bespoke aortic phantom was developed [11]. The phantom was designed to simulate the human aorta, able to deform with pulsatile and constant flow conditions, allowing for comparison of novel pressure drop estimation techniques to ground-truth pressure drop data from pressure sensors across a 3D-printed aortic valve.

A silicone 3D-printed aortic valve model was placed within a semi-compliant silicone tube, suspended in an acrylic box (Fig. 1). The valve was designed to resemble a healthy aortic valve, segmented from patient CT scans. The valve design was converted to a mould, before degassed Ecoflex 0030 silicone (Smooth-On Inc., Macungie, PE, USA) was poured in and left to cure. Valve mounts were also 3D printed using poly-lactic acid.

8 PendoTech pressure sensors (PRESS-S-000 sensor, PendoTech, Princeton, NJ, USA) were embedded along the tube wall, 1 situated before the valve and 7 downstream. The position of each pressure sensor was decided aiming to both capture the event of maximum constriction (i.e. location of the vena contracta) and the distal net pressure drop (i.e. characterisation of the pressure recovery). The sensors were calibrated and validated using a pressure catheter (MikroCath, Millar Inc, Houston, TX, USA). The pressure sensors were wired to input modules of a 
data acquisition USB chassis (National Instruments, Austin, TX, USA) to record ground-truth pressures for the 8 locations along the silicone tube at a sampling frequency of $10 \mathrm{KHz}$ (Fig. 2).

A $20 \mathrm{~L}$ external reservoir containing blood mimicking fluid [12] was connected to a CardioFlow 5000MR flow pump (Shelley Medical Imaging Technologies, Ontario, Canada). The pump was programmed, via control unit, to circulate approximately $15 \mathrm{~L}$ of blood mimicking fluid at eight different flow rates $(100,150,200$ and $250 \mathrm{~mL} / \mathrm{s}$, constant and pulsatile flows). These flow rates were programmed to generate maximum velocities of flow across the valve between 1.6 and $2.4 \mathrm{~m} / \mathrm{s}$ and pressure drops between 9.7 and $22.7 \mathrm{mmHg}$. Flow was maintained at a constant rate throughout each acquisition in the constant flow conditions. The pulsatile flow conditions were programmed to closely resemble the flow waveforms produced by the human heart, with fluctuations in pressure corresponding to systole and diastole.

\section{Velocity and ground truth pressure data acquisition and analysis}

Continuous wave Doppler and BSI data were acquired using a Vivid E95 ultrasound machine and 6S-D cardiac phased array transducer (GE Healthcare, Oslo, Norway) across the valve for each flow condition. Ground-truth pressure drop data were calculated using the methods outlined below. A metal clamp held the probe in position. The tilt angle between the tube and probe was recorded and used for angle correction calculations (Fig. 3). Each experimental condition was repeated on a second day of experiments.

Pressure data in pulsatile conditions was enhanced with a Butterworth filter that reduced the random peaks and noise on the raw temporal transients of pressure data from each sensor. For each experimental condition and flow rate, pressure data were recorded over 5 seconds and captured 4-5 cycles. The cycles were then segmented before the mean and standard deviation 
of the pressure transient, across 3 cycles, were calculated. The instant of peak pressure

117 difference between channels 1 and 2 or 3 was selected for further analysis. The mean and standard deviation values from each pressure sensor were then plotted against their physical position in the phantom, relative to the valve at point $0 \mathrm{~cm}$. Spline interpolation was performed between the valve and channel 4 , the region of the vena contracta, to estimate the maximum pressure drop and its location, relative to the valve (Fig. 4A). In constant flow conditions, a Butterworth filter was applied to the pressure signals before the mean and standard deviation values from each pressure sensor were then plotted against their physical position in the phantom. (Fig. 4B).

To estimate maximum velocity using Doppler, a continuous wave acquisition was acquired with the cursor placed at the valve opening. Once acquired, the E95 machine was used to manually select the maximum velocity observed in the acquisition (Fig. 5A). To estimate maximum velocity using BSI, the blood speckle imaging setting was used on the Vivid E95 machine to acquire BSI velocity data. A movie containing examples of BSI acquisitions at the different flow rates can be viewed in the additional files (Additional File 1). Offline analysis was performed to extract the maximum velocity observed across the acquired frames. Secondary validation was performed by an observer, using the interface shown in Fig. 5B to confirm that the maximum velocity vector was indeed observed within the expected region of interest where the jet after the valve is observed.

Pressure drop values were estimated from Doppler and BSI acquisitions by applying an angle correction, using the measured probe angle (Fig. 3), to the maximum velocity $(\mathrm{m} / \mathrm{s})$ value measured by each technique. Following this, the Bernoulli formulation was applied to the angle-corrected velocity value to convert to transvalvular pressure drop ( $\mathrm{mmHg}$ ). 
Pressure sensor data were extracted and analysed offline using MATLAB (Mathworks, Natick, MA, USA). To calibrate the pressure sensors for random error, the mean pressure across the 8 pressure sensors was calculated with static fluid in the phantom, before and after each experimental condition. For each condition, a correction was applied to each pressure sensor based on its deviation from this set mean. Pressure drop and flow velocity data are presented as the mean \pm standard deviation. To calculate the significance level of the values estimated by each technique, a paired two-tailed distribution t-test was used with a significance level of $\mathrm{p}<$ 0.05 (Microsoft Excel, Microsoft Office Version 2102, Microsoft Corporation, Redmond, Washington, USA).

\section{Results}

The probe angles were $24^{\circ}$ and $40^{\circ}$ on two days of experiments, respectively. The mean pressure drops across the valve, recorded by the ground-truth pressure sensors, were $9.75 \pm$ $0.07,12.4 \pm 0.00,16.4 \pm 0.21$ and $19.3 \pm 0.14 \mathrm{mmHg}$ under constant flow conditions and 11.6 $\pm 0.57,14.9 \pm 0.49,18.5 \pm 0.57$ and $23.6 \pm 1.23 \mathrm{mmHg}$ under pulsatile conditions, at the flow rates investigated $(100,150,200,250 \mathrm{~mL} / \mathrm{s}$, respectively). There was no significant difference between the mean pressure drop values acquired under constant vs. pulsatile conditions ( $\mathrm{p}>$ 0.05). The pressure drop values measured by the sensors were converted to velocity using:

$$
\text { Velocity }=\frac{\sqrt{\text { Pressure }} \text { Drop }}{4}
$$

The mean flow velocities across the valve were $1.6 \pm 0.0,1.8 \pm 0.0,2.0 \pm 0.0$ and $2.2 \pm 0.0 \mathrm{~m} / \mathrm{s}$ under constant flow conditions and $1.7 \pm 0.0,1.9 \pm 0.0,2.2 \pm 0.0$ and $2.4 \pm 0.1 \mathrm{~m} / \mathrm{s}$ under pulsatile conditions, at the flow rates investigated (100, 150,200, $250 \mathrm{~mL} / \mathrm{s}$, respectively). There was no significant difference between the mean velocity values acquired under constant vs. pulsatile conditions ( $\mathrm{p}>0.05)$. 
164 The pressure drop values estimated across the 4 flow rates by the ground-truth pressure sensors, 165 BSI and Doppler methods are presented in Fig. 6. No significant differences were observed between pressure drops estimated by Doppler, BSI and ground-truth sensors at the $100 \mathrm{~mL} / \mathrm{s}$ 167 pump flow rate $(10.4 \pm 1.76,10.3 \pm 1.63$ vs. $10.7 \pm 1.12 \mathrm{mmHg}$, respectively; $\mathrm{p}>0.05)$.

Nevertheless, under the 150, 200 and $250 \mathrm{~mL} / \mathrm{s}$ pump flow rates, pressure drops estimated by BSI $(10.5 \pm 1.47,13.7 \pm 2.27$ and $14.5 \pm 2.14 \mathrm{mmHg}$, respectively) were significantly lower than ground-truth pressure drops $(13.6 \pm 1.44,17.4 \pm 1.29$ and $20.1 \pm 1.78 \mathrm{mmHg}$, respectively; $\mathrm{p}<0.05)$. On the other hand, pressure drops estimated from Doppler velocity data were significantly higher than ground-truth pressure drop under $250 \mathrm{~mL} / \mathrm{s}$ pump flow conditions $(26.4 \pm 1.52$ vs. $20.1 \pm 1.78 \mathrm{mmHg}$, respectively; $\mathrm{p}<0.05)$.

The mean percentage errors in pressure drop estimation for each flow rate investigated, compared to the ground-truth pressure sensors, were $-2.26 \pm 17.7,13.8 \pm 22.2,17.4 \pm 12.3$ and $32.5 \pm 14.9 \%$ by Doppler and $-2.00 \pm 22.8,-22.5 \pm 8.01,-21.5 \pm 11.3$ and $-26.9 \pm 13.9 \%$ by BSI at the flow rates investigated $(100,150,200,250 \mathrm{~mL} / \mathrm{s}$, respectively; Fig. 7). The percentage error in pressure drop estimation by Doppler at the highest flow rate $(32.5 \pm 14.9$ $\%)$ was significantly greater than the percentage error at the lowest flow rate $(-2.26 \pm 17.7 \%$; $p<0.05)$.

Bland-Altman analysis of the Doppler and BSI techniques, compared to ground-truth pressure drop are presented in Fig. 8 [13]. Fig. 8A, shows that the bias of pressure drop estimations made using Doppler, when compared to ground-truth pressure drop was $3.54 \mathrm{mmHg}$. The range of the limits of agreement was $20.31 \mathrm{mmHg}$ (Fig. 8A). Fig. 8B shows that the bias of pressure drop estimations made using BSI, when compared to ground-truth pressure drop was -3.68 mmHg. The range of the limits of agreement was $13.2 \mathrm{mmHg}$ (Fig. 8B). 
Intra-technique reproducibility of the pressure drop estimations made across the two days of experiments by the three methods are presented in Fig. 9. Bland-Altman analysis produced intra-technique bias values of 0.56, 5.19 vs. $1.61 \mathrm{mmHg}$ for ground-truth, Doppler and BSI, respectively. The range of the limits of agreement within each technique were 2.40, 22.19 vs. $10.24 \mathrm{mmHg}$ for ground-truth, Doppler and BSI, respectively (Fig. 9).

\section{Discussion}

This study provides evidence to show that both BSI and Doppler techniques can make accurate estimations of low pressure drops in a controlled and reproducible aortic phantom. However, for stenotic conditions of clinical relevance in the setting of aortic stenosis, BSI underestimates while Doppler overestimates the pressure drop.

The assessment of the pressure drop by echocardiography in conventional clinical practice is subject to important methodological limitations that cannot be solved by current BSI technology. Significantly different pressure drop estimations, large percentage errors, bias values and wide limits of agreement exist for Doppler and BSI when compared with groundtruth pressure drop estimations. This is coupled with poor intra-technique reproducibility across two days of experiments. These findings illustrate that despite its theoretical advantages, further development of BSI or alternative novel and more comprehensive methods for pressure drop estimation are required to improve clinical practice.

\section{Pressure drop estimation}

A good agreement between pressure sensors, Doppler and BSI was found at low stenosis levels $(10.7 \pm 1.12 \mathrm{mmHg})$ but differences onset at the next stenotic condition tested $(13.6 \pm 1.44$ mmHg; Fig. 6). BSI would likely be accurate in the diastolic estimations of trans-mitral valve pressure drop and intra-cardiac pressure drops. When comparing Doppler and BSI to ground- 
truth pressure drop estimations, the limits of agreement are wide for both methods $(20.31$ mmHg for Doppler vs. $13.20 \mathrm{mmHg}$ for BSI; Fig. 8). Over/underestimations of transvalvular pressure drop by these margins are clinically significant.

213 At the higher pressure drops, with higher flow velocities, BSI significantly underestimates pressure drop (Fig. 6). A negative linear relationship is observed for percentage error (Fig. 7) and agreement (Fig. 8B), illustrating that this underestimation is more pronounced at higher flow rates. These results are consistent with previous findings conducted in vitro/in silico, whereby BSI was shown to underestimate flow velocity $[14,15,16]$. The largest in vivo study to date was performed in 51 healthy paediatric controls, where underestimations of velocity values acquired using BSI were also observed. The same study also revealed that the difference tended to increase at higher velocities [7]. The underestimation is most likely due to the current inability of the BSI algorithm to track these greater flow velocities, even in an optimal phantom configuration with low penetration depth (tracking is more challenging in deeper regions of interests).

On the other hand, pressure drops estimated using Doppler were significantly higher than ground-truth pressure drop at the greatest level of stenosis tested $(20.1 \pm 1.78 \mathrm{mmHg})$. This is likely due to the error in estimation of momentum from a single velocity value: the characterisation of the pressure drop requires the full velocity profile $[3,5]$. The bias of Doppler measurements across the experimental conditions was $3.54 \mathrm{mmHg}$; with peak overestimations of up to $18 \mathrm{mmHg}$ (Fig. 8A). These findings are consistent with those reported by Donati et al. (2017), where pressure drop values obtained using the Simplified Bernoulli formulation were shown to overestimate the true pressure drop by 54\% [4].

The peak pressure drop values measured by the pressure sensors in the phantom are different to net pressure drops measured in clinical practice during cardiac catheterisation, which 
measure the pressure difference between the left ventricular outflow tract and the ascending aorta, downstream of the vena contracta [17]. The peak pressure drop measured by the pressure sensors in the phantom is at the location of the vena contracta, as is the case for the Doppler data. The effect of pressure recovery further downstream, which is the traditional understanding of the reason of pressure drop overestimation by Doppler, can therefore be excluded.

The percentage error of measurements made using Doppler increased with flow rate and a significant difference in percentage error was observed between the highest and lowest flow rates (Fig. 7). A linear increase in the difference between the estimated pressure drop of Doppler vs. ground-truth with increasing level of stenosis can also be observed in the respective Bland-Alman agreement plot in Fig. 8A.

These findings support the observation that the overestimation of pressure drop by Doppler echocardiography is more pronounced at higher flow rates. The simplified Bernoulli formulation is accurate for uniform Doppler velocity profiles, observed at low flow velocities [4]. As flow rate and pressure drop increased in our experiments, the flow profiles became more heterogenous, explaining why Doppler is less accurate under these higher flow regimes

(Fig. 7). Further variability would be expected if different valve models and geometries were studied, or indeed if using in vivo data. These conditions would likely increase the degree of mismatch between true and estimated pressure drops further.

As a final minor remark, no significant difference was observed between the mean pressure drops achieved under constant and pulsatile conditions, allowing them to be grouped within each pump flow rate (Fig. 6; $n=4)$. 
The intra-technique reproducibility analysis demonstrates the strong reproducibility of the ground-truth pressure readings (Bias $0.56 \mathrm{mmHg}$; Fig. 9A). Variability is greater in the pulsatile estimations since each pressure drop is calculated as the mean over 3 cycles. BSI is less variable than Doppler (Bias 1.61 vs $5.19 \mathrm{mmHg}$, respectively; Fig. 9B-C). However, the reproducibility of BSI may be positively influenced by its inability to track high pressures, resulting in false clustering of measurements (Fig. 9C).

\section{Limitations}

These experiments were performed using a single model of a healthy aortic valve. The ultrasound probe was placed directly against the phantom to make the BSI and Doppler acquisitions with low penetration depth. These two factors represent a best-case scenario for the acquisition data for pressure drop estimation. In human subjects, acquisitions would be at an increased penetration depth, thus reducing the imaging frame rate, with an increased level of attenuation. In addition, experiments in human valves would exhibit more physiological and/or pathological variation. The results, therefore, would likely be different if data were obtained in vivo.

Future technological advances may improve the ability of BSI to track higher flow velocities and therefore estimate greater pressure drops more accurately. Future in vivo studies are required before BSI can be used in the clinical setting.

\section{Conclusions}

BSI accurately estimated pressure drops up to $10.7 \mathrm{mmHg}$ in controlled and reproducible phantom conditions of low stenotic burden, which may be useful for diastolic estimations of trans-mitral valve pressure drop and intra-cardiac pressure drops. BSI underestimated greater pressure drops, likely due to an inability of the algorithm to track higher flow velocities. 
Doppler overestimated greater pressure drops, in line with the published literature. Although

281

282

283

284

285

286

287

288

289

290

291

292

293

294

295

296

297

298

299

300

BSI offers a number of theoretical advantages to conventional Doppler echocardiography, further refinements and clinical studies are required with BSI before it can be used to improve transvalvular pressure drop estimation in the clinical evaluation of aortic stenosis.

\section{List of Abbreviations}

BSI - Blood speckle imaging

CT scan - Computerised tomography scan

\section{Figure Legends}

Fig. 1. The Ecoflex 0030 silicone aortic valve used for the experiments. Pictured from the front, outside of tube (left) and from the back, in situ (right).

Fig. 2. The phantom used for the velocity-based pressure drop estimation experiments.

Fig. 3. A photograph taken during the blood speckle imaging experiments, illustrating probe orientation and tilt angle measurement.

Fig. 4. Example plots of the pressure drop measured by the pressure sensors at $250 \mathrm{~mL} / \mathrm{s} \mathrm{A}$ ) pulsatile flow B) constant flow rates. Blue points and error bars represent mean \pm standard deviation from the 8 pressure sensors. The red point represents the maximum pressure drop and its location, estimated using spline interpolation between the valve and sensor 4 . 
Fig. 5. A) Example continuous wave doppler acquisition at $250 \mathrm{~mL} / \mathrm{s}$ flow rate with manual measurement shown. B) Example BSI velocity vector interface, showing the velocity of flow through the region of interest.

Fig. 6. Angle-corrected pressure drop (Bernoulli equation; mmHg) from BSI (grey dotted bars) and Doppler (light grey bars), compared to ground truth acquired by sensors (dark grey bars). Data are presented as the mean estimated drop under each flow condition, grouping constant and pulsatile flows. Error bars represent \pm standard deviation.* denotes statistical significance $(\mathrm{p}<0.05)$.

Fig. 7. Percentage error of pressure drop estimations (\%) from BSI (grey dotted bars) and Doppler (light grey bars), compared to ground truth acquired by sensors. Data are presented as the mean value (cross), the median value (line), the upper and lower quartiles (box range) and the minimum and maximum values (whiskers) under each flow condition, grouping constant and pulsatile flows. * denotes statistical significance compared to the $100 \mathrm{~mL} / \mathrm{s}$ flow rate $(\mathrm{p}<0.05)$.

Fig. 8. Bland-Altman plots illustrating the agreement between A) Doppler vs. ground-truth and B) BSI vs. ground-truth pressure drop estimations $(n=16)$. The solid line represents the bias between the 2 methods, while the $95 \%$ limits of agreement ( \pm 1.96 standard deviation) are represented by the dashed lines. 
Fig. 9. Bland-Altman plots illustrating the agreement between A) ground-truth B) Doppler and C) Blood Speckle Imaging estimations of pressure drop across two days of experiments $(n=16)$. The solid line represents the bias within the methods, while the $95 \%$ limits of

327 agreement ( \pm 1.96 standard deviation) are represented by the dashed lines.

\section{$328 \quad$ Additional Files}

329 File name: Additional File 1

330 File type: WMV file (.wmv)

331 Title of data: Example BSI Acquisitions

332 Description: A video file containing examples of the BSI acquisitions at each of the flow

333 rates studied

\section{Declarations}

335 Ethics approval and consent to participate

336 Not applicable.

337 Consent for Publication

338 Not applicable.

339 Availability of data and materials

340 The datasets used and/or analysed during the current study are available from the

341 corresponding author upon reasonable request.

342 Competing interests

343 The authors have no competing interests to declare. 
C. Dockerill was supported by the UK Medical Research Council [MR/N013700/1] and the

346 King's College London MRC Doctoral Training Partnership in Biomedical Sciences. This

347 work was also supported by the British Heart Foundation [TG/17/3/33406], by the European Union's Horizon 2020 research and Innovation programme under the Marie Sklodowska-Curie grant agreement [No 764738], and by the Wellcome EPSRC Centre for Medical Engineering at King's College London [WT 203148/Z/16/Z]. P. Lamata holds a Wellcome Trust Senior Research Fellowship [209450/Z/17/Z].

\section{Authors' contributions}

353 All authors contributed to the study design and interpretation of results. HG, JF, AN and CD

354 were involved in phantom design and construction. HG, CD and AN performed data

355 acquisition. AN and CD performed data analysis. CD and PL drafted the manuscript. The 356 final version was reviewed by all authors and investigators.

\section{Acknowledgements}

358 The authors would like to thank Dr Olivier Gerard and his team at GE Healthcare for their 359 guidance and expertise on acquiring the BSI data.

\section{References}

[1] Currie PJ, Seward JB, Reeder GS, et al. Continuous-wave Doppler echocardiographic assessment of severity of calcific aortic stenosis: a simultaneous Doppler-catheter correlative study in 100 adult patients. Circulation. 1985;71(6):1162-1169. 
[2] Nishimura RA, Carabello BA. Hemodynamics in the cardiac catheterization laboratory of the

$21 \mathrm{st}$

century.

Circulation.

2012;125(17):2138-2150.

[3] Donati F, Myerson S, Bissell MM, et al. Beyond Bernoulli: Improving the Accuracy and Precision of Noninvasive Estimation of Peak Pressure Drops. Circ Cardiovasc Imaging. 2017;10(1):e005207. doi:10.1161/CIRCIMAGING.116.005207

[4] Ring L, Shah BN, Bhattacharyya S, et al. Echocardiographic assessment of aortic stenosis: a practical guideline from the British Society of Echocardiography. Echo Res Pract. 2021;8(1):G19-G59. Published 2021 Apr 28. doi:10.1530/ERP-20-0035

[5] Gill H, Fernandes J, Chehab O, et al. Evaluation of aortic stenosis: From Bernoulli and Doppler to Navier-Stokes [published online ahead of print, 2021 Dec 15]. Trends Cardiovasc Med. 2021;S1050-1738(21)00146-8. doi:10.1016/j.tcm.2021.12.003

[6] Trahey GE, Allison JW, von Ramm OT. Angle independent ultrasonic detection of blood flow. IEEE Trans Biomed Eng. 1987;34(12):965-967. doi:10.1109/tbme.1987.325938

[7] Hansen KL, Nielsen MB, Jensen JA. Vector velocity estimation of blood flow - A new application in medical ultrasound. Ultrasound. 2017;25(4):189-199. doi:10.1177/1742271X17713353

[8] Nyrnes SA, Fadnes S, Wigen MS, Mertens L, Lovstakken L. Blood Speckle-Tracking Based on High-Frame Rate Ultrasound Imaging in Pediatric Cardiology. J Am Soc Echocardiogr. 2020;33(4):493-503.e5. doi:10.1016/j.echo.2019.11.003

[9] Geyer H, Caracciolo G, Abe H, et al. Assessment of myocardial mechanics using speckle tracking echocardiography: fundamentals and clinical applications [published correction appears in J Am Soc Echocardiogr. 2010 Jul;23(7):734]. J Am Soc Echocardiogr. 2010;23(4):351-455. doi:10.1016/j.echo.2010.02.015 
[10] Fadnes S, Nyrnes SA, Torp H, Lovstakken L. Shunt flow evaluation in congenital heart disease based on two-dimensional speckle tracking. Ultrasound Med Biol. 2014;40(10):2379-2391. doi:10.1016/j.ultrasmedbio.2014.03.029

[11] Gill H, Fernandes JF, Bissell M et al. 3D printed valve models replicate in vivo bicuspid aortic valve peak pressure drops. J Am Coll Cardiol. 2020;75(11) Supplement_1.

[12] Ramnarine KV, Nassiri DK, Hoskins PR, Lubbers J. Validation of a new blood-mimicking fluid for use in Doppler flow test objects. Ultrasound Med Biol. 1998;24(3):451-459. doi:10.1016/s0301-5629(97)00277-9

[13] Bland JM, Altman DG. Statistical methods for assessing agreement between two methods of clinical measurement. Lancet. 1986;1(8476):307-310.

[14] Van Cauwenberge J, Lovstakken L, Fadnes S, et al. Assessing the Performance of Ultrafast Vector Flow Imaging in the Neonatal Heart via Multiphysics Modeling and In Vitro Experiments. IEEE Trans Ultrason Ferroelectr Freq Control. 2016;63(11):1772-1785. doi:10.1109/TUFFC.2016.2596804

[15] Fadnes S, Wigen MS, Nyrnes SA, Lovstakken L. In Vivo Intracardiac Vector Flow Imaging Using Phased Array Transducers for Pediatric Cardiology. IEEE Trans Ultrason Ferroelectr Freq Control. 2017;64(9):1318-1326. doi:10.1109/TUFFC.2017.2689799

[16] Wigen MS, Fadnes S, Rodriguez-Molares A, et al. 4-D Intracardiac Ultrasound Vector Flow Imaging-Feasibility and Comparison to Phase-Contrast MRI. IEEE Trans Med Imaging. 2018;37(12):2619-2629. doi:10.1109/TMI.2018.2844552

[17] Garcia D, Pibarot P, Dumesnil JG, Sakr F, Durand LG. Assessment of aortic valve stenosis severity: A new index based on the energy loss concept. Circulation. 2000;101(7):765-771. doi:10.1161/01.cir.101.7.765 


\section{Figures}

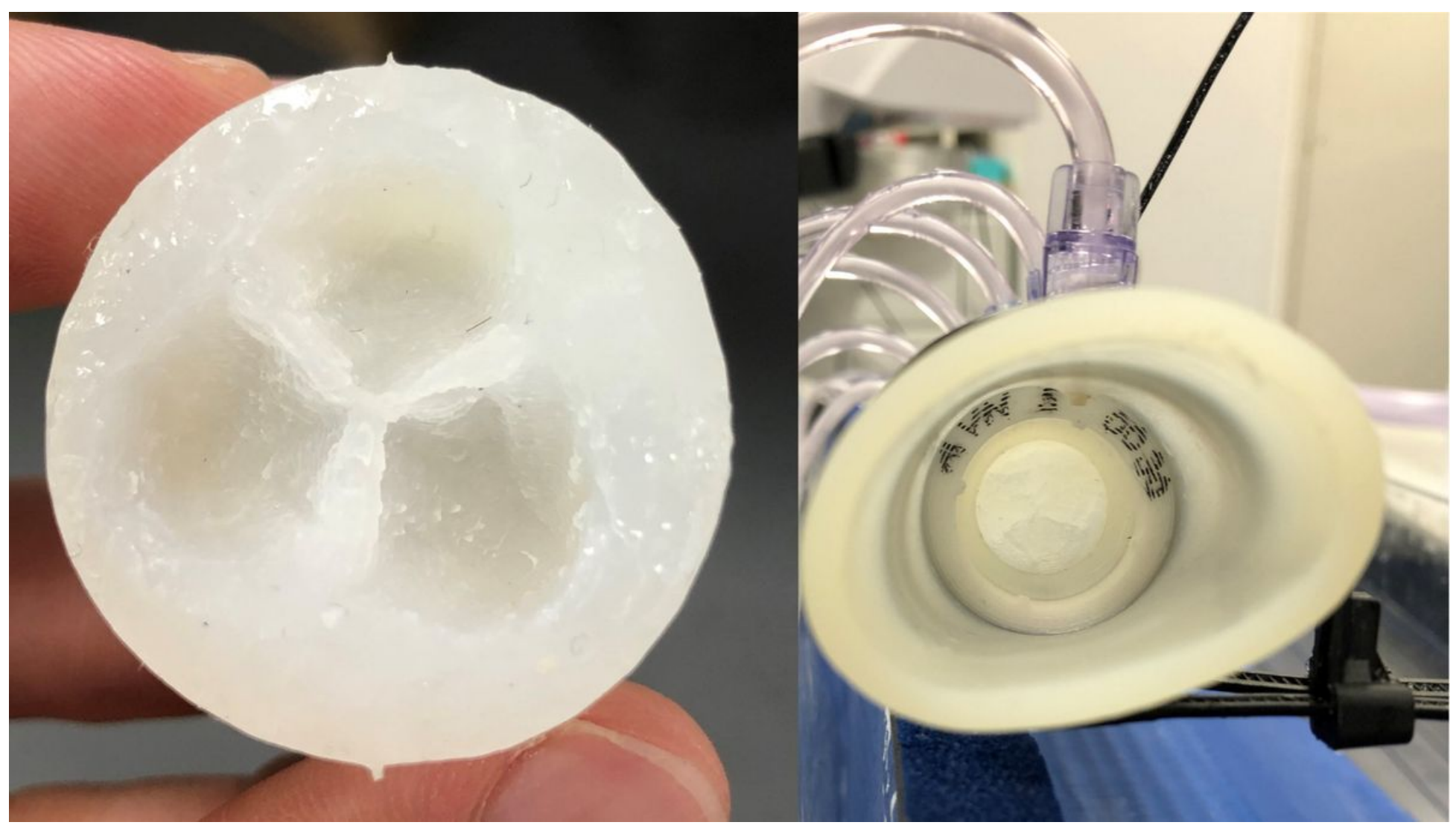

\section{Figure 1}

The Ecoflex 0030 silicone aortic valve used for the experiments. Pictured from the front, outside of tube (left) and from the back, in situ (right).

\section{Figure 2}

The phantom used for the velocity-based pressure drop estimation experiments. 


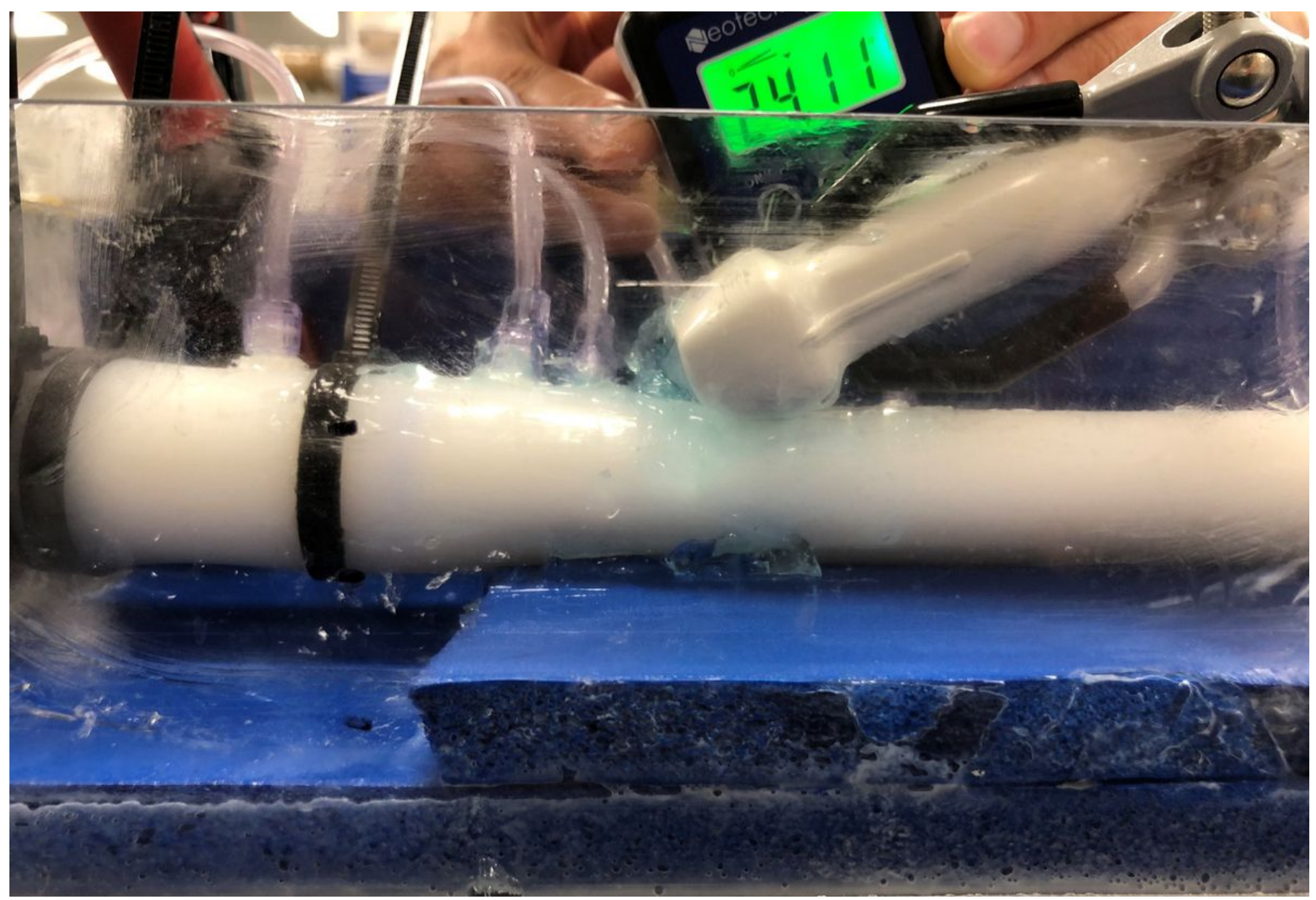

Figure 3

A photograph taken during the blood speckle imaging experiments, illustrating probe orientation and tilt angle measurement.
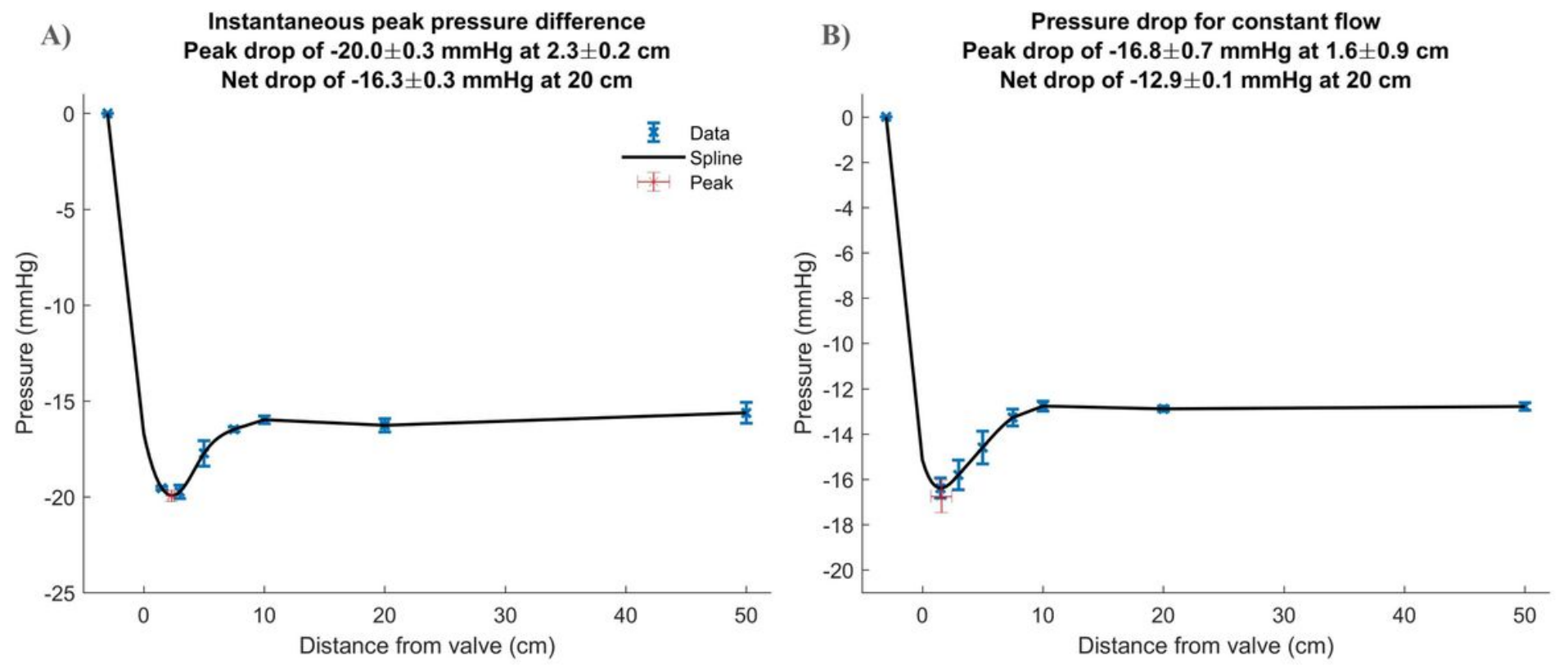


\section{Figure 4}

Example plots of the pressure drop measured by the pressure sensors at $250 \mathrm{~mL} / \mathrm{s} \mathrm{A}$ ) pulsatile flow B) constant flow rates. Blue points and error bars represent mean \pm standard deviation from the 8 pressure sensors. The red point represents the maximum pressure drop and its location, estimated using spline interpolation between the valve and sensor 4 .

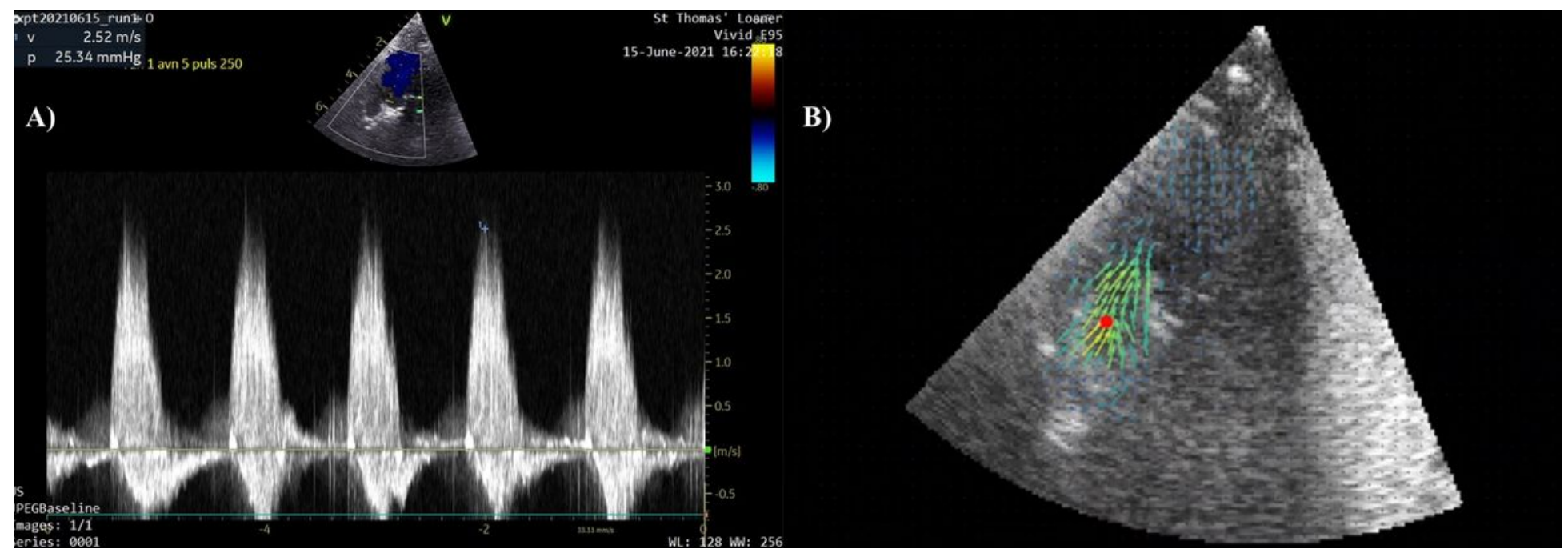

\section{Figure 5}

A) Example continuous wave doppler acquisition at $250 \mathrm{~mL} / \mathrm{s}$ flow rate with manual measurement shown. B) Example BSI velocity vector interface, showing the velocity of flow through the region of interest. 


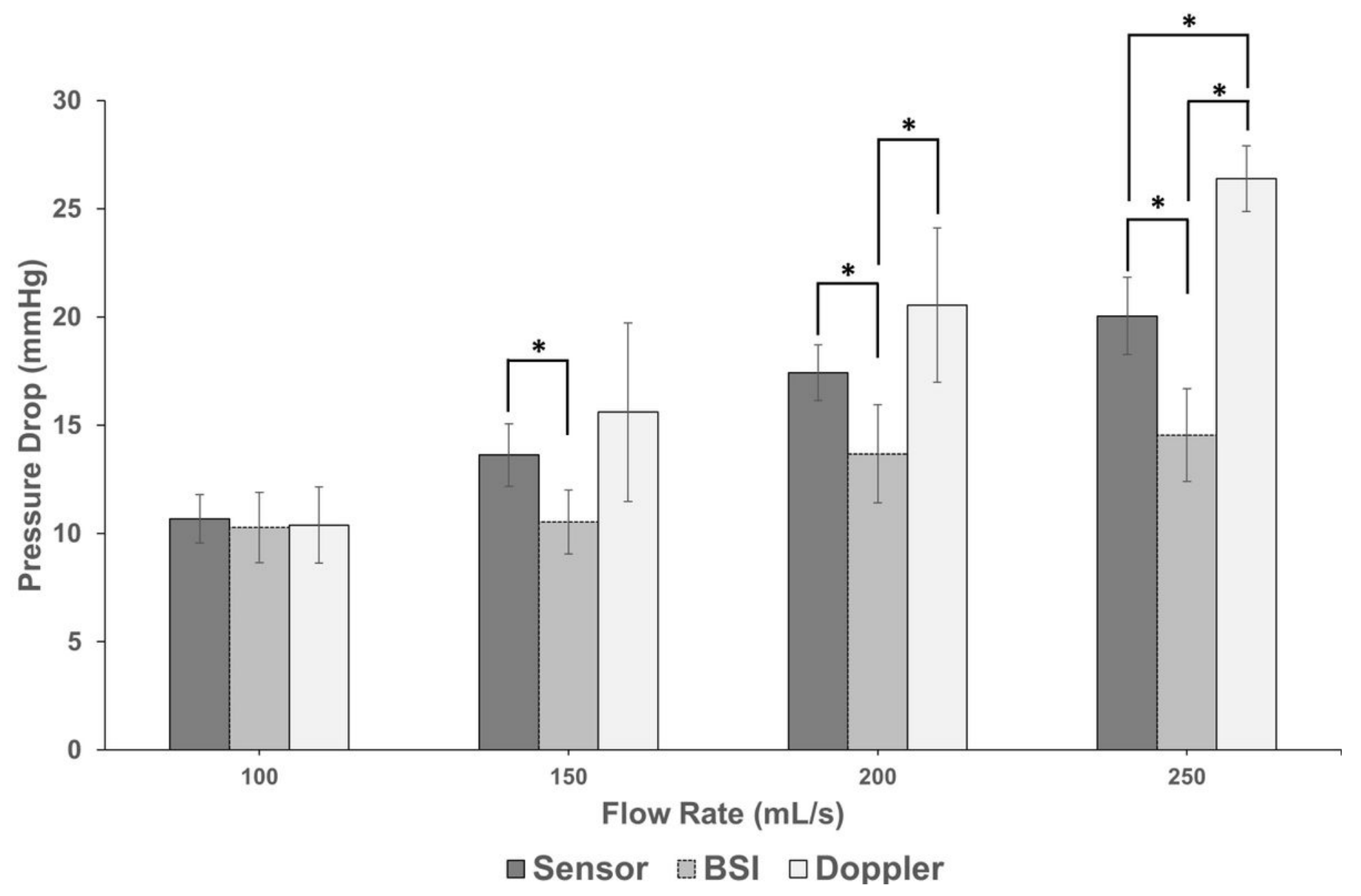

Figure 6

Angle-corrected pressure drop (Bernoulli equation; mmHg) from BSI (grey dotted bars) and Doppler (light grey bars), compared to ground truth acquired by sensors (dark grey bars). Data are presented as the mean estimated drop under each flow condition, grouping constant and pulsatile flows. Error bars represent \pm standard deviation. ${ }^{*}$ denotes statistical significance $(p<0.05)$. 


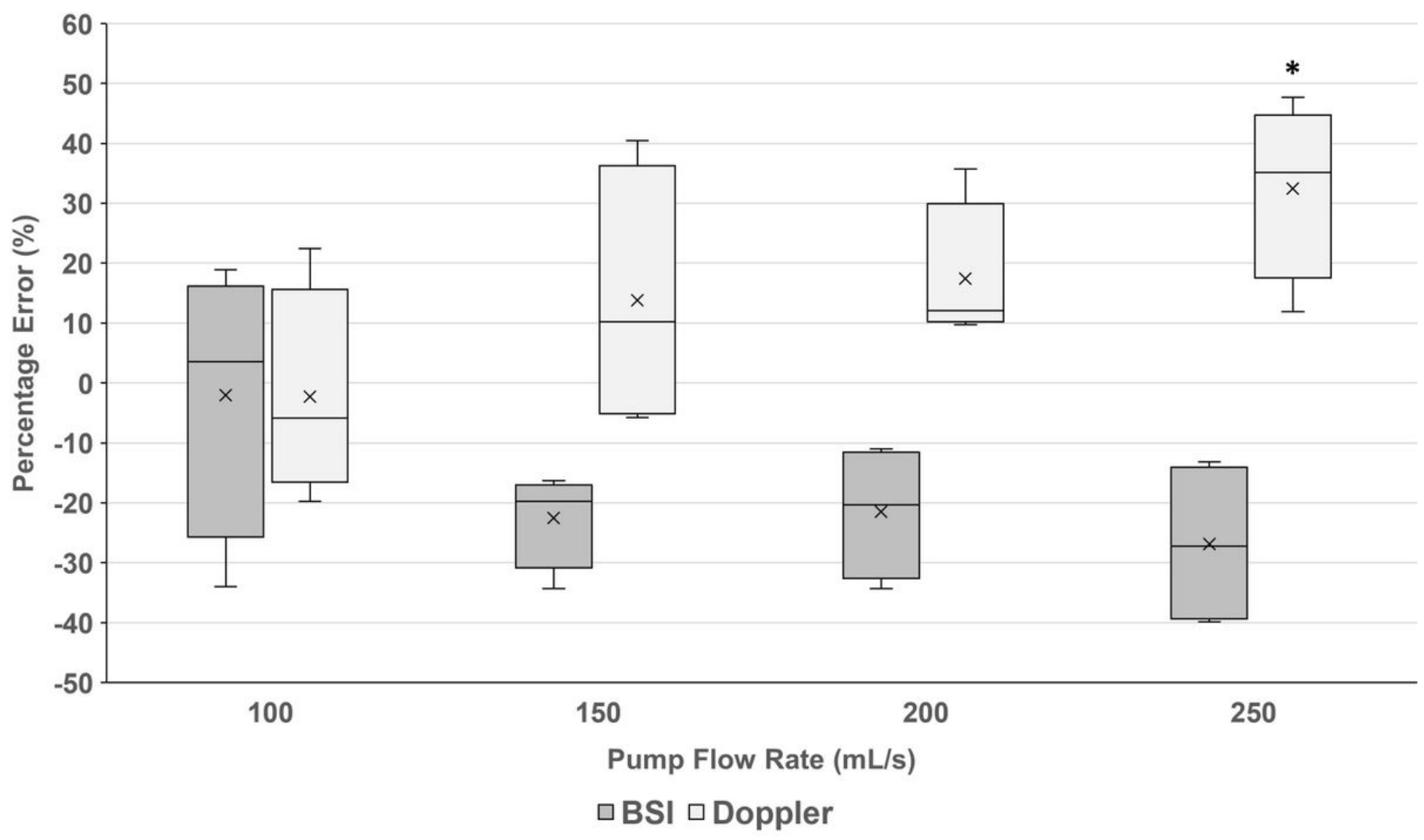

Figure 7

Percentage error of pressure drop estimations (\%) from BSI (grey dotted bars) and Doppler (light grey bars), compared to ground truth acquired by sensors. Data are presented as the mean value (cross), the median value (line), the upper and lower quartiles (box range) and the minimum and maximum values (whiskers) under each flow condition, grouping constant and pulsatile flows. * denotes statistical significance compared to the $100 \mathrm{~mL} / \mathrm{s}$ flow rate $(p<0.05)$.
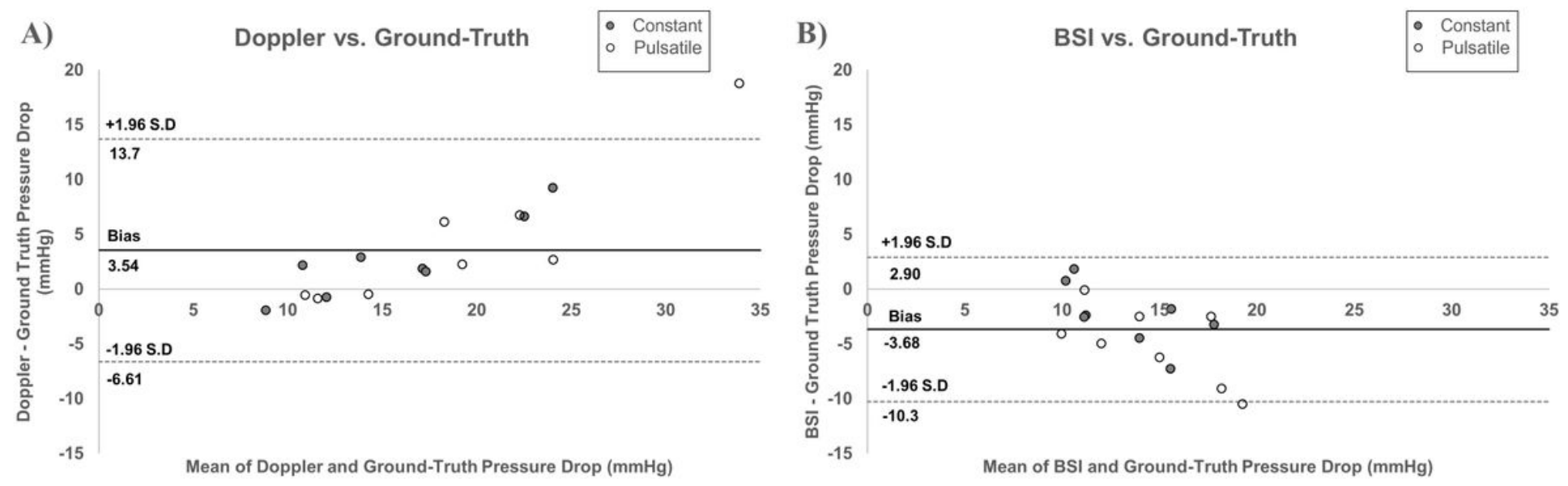

\section{Figure 8}


Bland-Altman plots illustrating the agreement between A) Doppler vs. ground-truth and B) BSI vs. groundtruth pressure drop estimations $(n=16)$. The solid line represents the bias between the 2 methods, while the $95 \%$ limits of agreement ( \pm 1.96 standard deviation) are represented by the dashed lines.
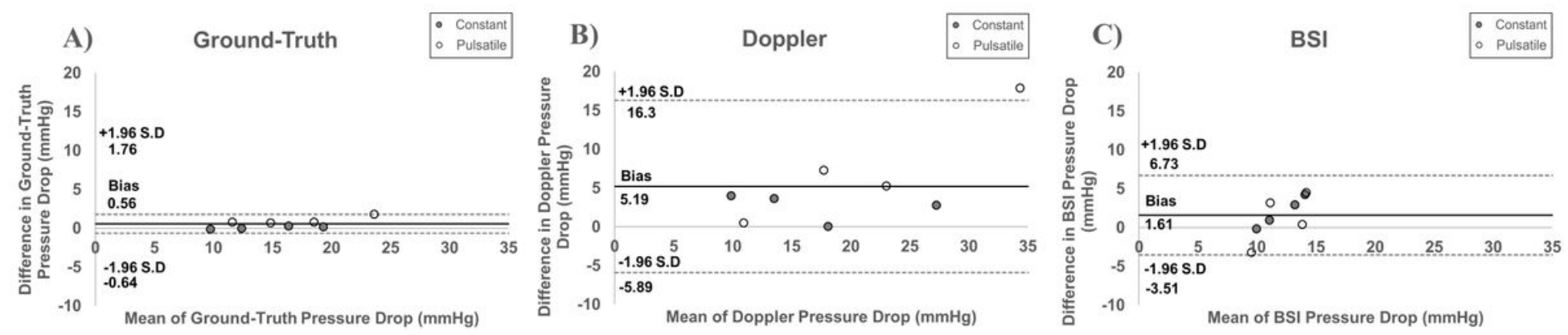

Figure 9

Bland-Altman plots illustrating the agreement between A) ground-truth B) Doppler and C) Blood Speckle Imaging estimations of pressure drop across two days of experiments $(n=16)$. The solid line represents the bias within the methods, while the $95 \%$ limits of agreement ( \pm 1.96 standard deviation) are represented by the dashed lines.

\section{Supplementary Files}

This is a list of supplementary files associated with this preprint. Click to download.

- BSIClips.wmv 